\title{
Morphine and the blood-brain barrier: diffusion, uptake, or efflux?
}

\author{
Karsten Bartels, MD $\cdot$ Konrad Meissner, MD
}

Received: 16 June 2017/Revised: 20 June 2017/ Accepted: 5 July 2017/Published online: 18 July 2017

(C) Canadian Anesthesiologists' Society 2017

In order to balance a strictly regulated microenvironment, the central nervous system (CNS) is separated from the blood by three barriers: the blood-brain barrier, the bloodcerebrospinal fluid barrier, and the arachnoid barrier. ${ }^{1}$ As the primary transfer interface between blood and the CNS, the blood-brain barrier maintains a tightly regulated milieu to permit undisturbed signalling between the cells of the CNS. Substances arriving at the blood-brain barrier, be they toxic or therapeutic, face three distinct mechanisms that regulate barrier function: first, a physical barrier made up of the tight junctions between endothelial cells; second, a transport barrier made up of specific membrane-bound transport proteins on both the luminal and abluminal side of the membrane; and third, a metabolic barrier consisting of intra- and extracellular enzymes (Figure). ${ }^{2}$ The transport proteins form a biochemically selective barrier that relies on the dynamic integration of neuronal, glial, and vascular components, thus comprising a neurovascular unit. ${ }^{3,4}$ Altered blood-brain barrier function in the setting of pathologic states can lead to increased exposure of the brain to various neurotoxins.

Animal models have provided the basis for much of our understanding of the changes induced in blood-brain barrier function during the perioperative period. Most rigorously, the blood-brain barrier has been studied in the context of stroke and neuroinflammatory disorders.

K. Bartels, MD $(\bowtie)$

Department of Anesthesiology, University of Colorado Denver, 12401 E. 17th Avenue, Leprino Office Building, 7th Floor, MS

B-113, Aurora, CO 80045, USA

e-mail: karsten.bartels@ucdenver.edu

K. Meissner, MD

Klinik für Anästhesiologie, Universitätsmedizin Greifswald, Greifswald, Germany
Membrane proteins of the adenosine triphosphate (ATP)binding cassette $(\mathrm{ABC})$ transporter family protect the CNS against potentially toxic exogenous substances by actively removing them from the brain. In so doing, they control drug and metabolite transport to and from the brain. The $\mathrm{ABC}$ transporters are distinctly regulated in CNS models of ischemia and inflammation and include, among others, the efflux transporters P-glycoprotein (PGP), multidrug resistance proteins (MRPs), and breast cancer resistance protein (BCRP). ${ }^{4,5}$ In addition to these efflux transporters, it is also evident that uptake transporters, such as organic anion-transporting polypeptides (e.g., OATP1A2) and organic cation transporters (e.g., OCT1) are expressed at the blood-brain barrier. ${ }^{6,7}$ Net movement of substances into the CNS reflects a product of active uptake and efflux across the blood-brain barrier. Genetic variants of both OCT1 and MRP3 have been associated with altered postoperative effects of morphine in humans. Such changes are readily explainable by systemic (non-bloodbrain barrier) effects, as expression of these transport proteins is not limited only to the brain. 8

The clinical relevance for the discovery of specific substrates for human blood-brain barrier transporters is their potentially variable compartment concentrations based on genetically variable expression of their respective transport proteins. To date, the lack of specific inhibitors approved for such use in humans remains a major challenge for clinical studies examining precise roles of individual ABC transporters. Nonetheless, the effects of morphine in human volunteers were enhanced by the coadministration of the PGP inhibitor cyclosporine. ${ }^{10}$ Still, functional evidence for physiologically altered levels of PGP expression in humans remains uncertain.

While results within different models vary, ${ }^{11}$ it appears that, following short-term exposure to inflammatory 


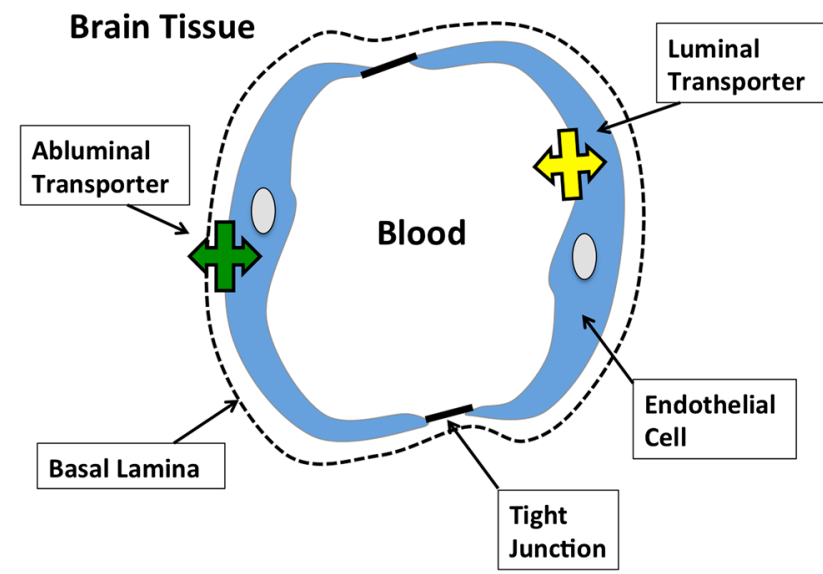

Figure Select mechanisms of blood-brain barrier function. Endothelial cells (blue) are enclosed by a basal lamina (dashed line) and connected via tight junction proteins to form a physical barrier between blood and brain tissue. Proteins performing active efflux or uptake of substances from or into the brain are embedded in the luminal (yellow) and abluminal (green) side of the endothelium and comprise the transport barrier ${ }^{2}$

Mécanismes choisis de la fonction de la barrière hématoencéphalique. Les cellules endothéliales (bleu) sont enfermées par une membrane basale (ligne pointillée) et connectées par les protéines de jonction serrée afin de former une barrière physique entre le sang et le tissu cérébral. Les protéines réalisant un efflux ou une absorption actifs de substances à partir du cerveau ou hors de ce dernier sont encastrées dans les côtés luminaux (jaune) et abluminaux (verts) de l'endothélium et comprennent la barrière de transport ${ }^{2}$

stimuli, such as tumor necrosis factor (TNF)- $\alpha$, PGP undergoes down-regulation mediated by activation of inducible nitric oxide synthase and up-regulation with long-term exposure to TNF- $\alpha .{ }^{12}$ The PGP and BCRP expression levels also increase after ischemia/reperfusion injury in rats, and peak expression correlates with behavioural recuperation. ${ }^{13}$ Paracellular permeability increases in response to hypoxia, an effect that is attenuated during reperfusion by increased expression of tight junction protein and up-regulation of luminal and abluminal transport proteins. ${ }^{14}$

In this issue of the Journal, Wang et al. report the results of a study examining the pharmacokinetics of morphine and its metabolites morphine-3-glucuronide (M3G) and morphine-6-glucuronide (M6G) in plasma and cerebrospinal fluid. ${ }^{15}$ Elegantly, the authors chose to study patients undergoing major open or endovascular thoracic aortic aneurysm repair, which enabled simultaneous measurement of morphine (and its metabolites) as well as pro-inflammatory cytokines and albumin in both cerebrospinal fluid (CSF) and plasma. Parsimoniously including open $v s$ endovascular performed aortic repairs allowed the authors access to two very different levels of perioperatively induced inflammation. Indeed, a differential pro-inflammatory effect was detected from the cytokine responses. The CSF/plasma area under the curve (AUC) ratios for morphine were not correlated with levels of the pro-inflammatory cytokine interlukin-6 (IL-6). Although a moderate positive correlation was detected between the M3G AUC CSF/plasma ratio and CSF IL-6, a surgery-specific effect of PGP on blood-brain barrier morphine efflux transport was not ascertained.

The authors should be congratulated on their ambitious work, which comprises one of the first attempts to quantify changes in transport barrier effects during a surgical stimulus. The authors' achievement is not diminished by the limitations of their work. For example, because of the potential differential expression of $\mathrm{ABC}$ transporters at the different compartments of the blood-brain barrier, ${ }^{12}$ their substrate specificity, as well as other unknown regulatory effects, their study results should be seen as specific for the chosen inflammatory stimulus and for opioid excretion into the CSF. Their observations probably represent the net effect of complex regulatory processes, which are likely impossible to decipher fully in human subjects. There are examples of $\mathrm{ABC}$ transport studies suggesting that in vitro and animal studies may overestimate the clinical relevance of $\mathrm{ABC}$ transport activity in humans. ${ }^{16-18}$ In light of known genetic variability of many $\mathrm{ABC}$ transporters in humans, the "negative" results reported in this issue of the Journal may, therefore, reassure clinicians. Nevertheless, tissue concentrations and opioid effects were not studied in this setting, even though morphine dosages were recorded.

Studying such performance in a real-world scenarioi.e., the three components of blood-brain barrier function (i.e., physical, transport, and metabolic) and the differential effects of blood-brain $v s$ blood-CSF barrier contributionswill always be subject to the inherent limitations of research in human subjects. Nevertheless, the active efflux and uptake of exogenous and endogenous substances play a critical role in maintaining CNS hemostasis in the face of a relatively preserved physical barrier. Therefore, further clinical studies to advance our understanding of perioperative alterations in select drug and toxin permeability across the blood-brain barrier should be encouraged.

\section{La morphine et la barrière hémato-encéphalique : diffusion, absorption / recaptage ou efflux?}

Afin d'équilibrer un micro-environnement strictement régulé, trois barrières séparent le système nerveux central (SNC) du sang: la barrière hémato-encéphalique, la barrière sang-liquide céphalorachidien, et la barrière 
arachnoïdienne. ${ }^{1}$ En tant que principale interface de transfert entre le sang et le SNC, la barrière hémato-encéphalique maintient un milieu extrêmement régulé afin de permettre une signalisation ininterrompue entre les cellules du SNC. Les substances qui parviennent à la barrière hémato-encéphalique, qu'elles soient toxiques ou thérapeutiques, font face à trois mécanismes distincts qui régulent la fonction de la barrière : en premier lieu, une barrière physique, composée des jonctions serrées entre les cellules endothéliales; deuxièmement, une barrière de transport, composée de protéines de transport spécifiques liées à la membrane sur les faces luminales et abluminales de la membrane; et, troisièmement, une barrière métabolique, composée d'enzymes intra- et extracellulaires (Figure). ${ }^{2}$ Les protéines de transport forment une barrière sélective d'un point de vue biochimique qui se fonde sur l'intégration dynamique de composantes neuronales, gliales et vasculaires, créant ainsi une unité neurovasculaire. ${ }^{3,4}$ Une fonction altérée de la barrière hémato-encéphalique provoquée par un état pathologique peut entraîner une exposition accrue du cerveau à diverses neurotoxines.

C'est sur la base de modèles animaux que se fonde une grande partie de notre compréhension des changements induits dans la fonction de la barrière hémato-encéphalique en période périopératoire. Les études les plus rigoureuses portant sur la barrière hémato-encéphalique ont été réalisées dans des contextes d'accidents vasculaires cérébraux et de troubles neuro-inflammatoires. Les protéines transmembranaires de la famille des transporteurs ABC (adenosine triphosphate (ATP) binding cassette) protègent le SNC des substances exogènes potentiellement toxiques en les extrayant activement du cerveau. Ce faisant, elles contrôlent le transport des médicaments et des métabolites vers le cerveau et hors de ce dernier. Les transporteurs ABC sont régulés de façon distincte dans les modèles ischémiques et inflammatoires du SNC et incluent, entre autres, la glycoprotéine $\mathrm{P}$ (PGP), un transporteur sortant, les protéines de résistance pléiotrope (MRP) et la protéine de résistance au cancer du sein (BCRP). ${ }^{4,5}$ Outre ces transporteurs sortants, il est également évident que des transporteurs d'absorption, tels les polypeptides transporteurs des anions organiques (par ex. le OATP1A2) et les transporteurs de cations organiques (par ex., le OCT1) s'expriment à la barrière hémato-encéphalique. $^{6,7}$ Les mouvements nets de substances dans le SNC reflètent des produits d'absorption et d'efflux actifs à travers la barrière hémato-encéphalique. Des variantes génétiques de l'OCT1 et de la MRP3 ont été associées à des effets postopératoires altérés de la morphine chez l'humain. De tels changements s'expliquent facilement par les effets systémiques (c.-à-d. non liés à la barrière hémato-encéphalique), étant donné que l'expression de ces protéines de transport ne se limite pas au cerveau. ${ }^{8,9}$

La pertinence clinique motivant la découverte de substrats spécifiques des transporteurs humains de la barrière hémato-encéphalique est leurs concentrations compartimentées potentiellement variables selon l'expression génétiquement variable de leurs protéines de transport respectives. À ce jour, l'absence d'inhibiteurs spécifiques approuvés pour un tel emploi chez l'humain constitue encore et toujours un défi majeur pour les études cliniques qui souhaitent examiner les rôles précis de transporteurs ABC individuels. Toutefois, on a observé que les effets de la morphine sur les volontaires humains étaient exacerbés par l'administration concomitante d'un inhibiteur de la PGP, la cyclosporine. ${ }^{10}$ Les données probantes fonctionnelles attestant de niveaux physiologiquement altérés de l'expression de la PGP demeurent toutefois peu concluantes.

Bien que les résultats varient d'un modèle à un autre, ${ }^{11}$ il semble que, après une brève exposition à des stimuli inflammatoires tels que le facteur onconécrosant $\alpha$ (tumor necrosis factor $\alpha-$ TNF- $\alpha$ ), la PGP subit une insensibilisation médiée par l'activation de la synthase inductible du monoxyde d'azote et une régulation à la hausse avec une exposition à long terme au TNF- $\alpha .^{12}$ Les taux d'expression de la PGP et de la BCRP augmentent également après une lésion d'ischémie-reperfusion chez le rat, et l'expression maximale est corrélée à la récupération comportementale. $^{13}$ La perméabilité paracellulaire augmente en réponse à l'hypoxie, un effet atténué pendant la reperfusion par une expression accrue de la protéine des jonctions serrées et une régulation à la hausse des protéines de transport luminales et abluminales. ${ }^{14}$

Dans ce numéro du Journal, Wang et coll. rapportent les résultats de leur étude, qui a examiné la pharmacocinétique de la morphine et de ses métabolites glucuronide M3G (morphimorphine-3-glucuronide) et glucuronide M6G (morphine-6-glucuronide) dans le plasma et le liquide céphalorachidien. $^{15}$ De façon élégante, les auteurs ont choisi d'étudier des patients subissant une réparation d'anévrisme aortique thoracique par thoracotomie ou par voie endovasculaire, ce qui a permis de mesurer simultanément la morphine (et ses métabolites) et les cytokines pro-inflammatoires et l'albumine dans le liquide céphalorachidien (LCR) et dans le plasma. En incluant des réparations aortiques invasives ou endovasculaires, les auteurs ont eu accès à deux niveaux différents d'inflammation induite par l'opération. En effet, un effet pro-inflammatoire différentiel a été détecté grâce aux réactions des cytokines. Les taux de surface sous la courbe (SSC) LCR/plasma pour la morphine n'étaient pas corrélés aux niveaux de cytokine pro-inflammatoire interleukine 6 (IL-6). Bien qu'une corrélation positive modérée ait été 
observée entre le taux de SSC du LCR/plasma de M3G et l'IL-6 dans le LCR, un effet spécifique à la chirurgie de la PGP sur le transport sortant de la morphine par la barrière hémato-encéphalique n'a pas été clairement établi.

Il faut féliciter les auteurs pour leur travail ambitieux : il s'agit de l'une des premières tentatives de quantifier les changements d'effets des transporteurs à travers la barrière pendant un stimulus chirurgical. Les prouesses des auteurs ne sont en rien diminuées par les limites de leur travail. Par exemple, en raison de l'expression différentielle potentielle des transporteurs $\mathrm{ABC}$ dans les divers compartiments de la barrière hémato-encéphalique, ${ }^{12}$ leur spécificité de substrat ainsi que d'autres effets régulateurs inconnus, les résultats de l'étude devraient être considérés comme étant spécifiques au stimulus inflammatoire choisi et à l'excrétion opioïde dans le LCR. Leurs observations représentent probablement l'effet net de processus de régulation complexes, lesquels sont probablement impossibles à déchiffrer intégralement chez l'humain. Il existe quelques études sur le transport $\mathrm{ABC}$ suggérant que les études in vitro et animales pourraient surestimer la pertinence clinique de l'activité de transport $\mathrm{ABC}$ chez l'humain. ${ }^{16-18}$ À la lumière de la variabilité génétique connue de plusieurs transporteurs ABC chez l'humain, les résultats « négatifs » rapportés dans ce numéro du Journal pourraient par conséquent rassurer les cliniciens. Toutefois, les concentrations tissulaires et les effets opioïdes n'ont pas été étudiés dans ce contexte, bien que les posologies de morphine aient été enregistrées.

L'étude d'une telle performance dans un contexte réel - c.-à-d. les trois composantes de la fonction de la barrière hémato-encéphalique (soit physique, de transport et métabolique) et les effets différentiels des contributions des barrières hémato-encéphalique vs hémato-LCR - sera toujours sujette aux limites inhérentes à la recherche chez l'humain. Toutefois, l'efflux et l'absorption actifs de substances exogènes et endogènes jouent un rôle crucial dans le maintien de l'hémostase du SNC face à une barrière physique relativement préservée. C'est pourquoi il faut encourager la réalisation d'autres études cliniques qui permettront de faire progresser notre compréhension des altérations périopératoires de perméabilité de certains médicaments et toxines à travers la barrière hémato-encéphalique.

\section{Conflicts of interest None declared.}

Editorial responsibility This submission was handled by Dr. Hilary P. Grocott, Editor-in-Chief, Canadian Journal of Anesthesia.

Funding Karsten Bartels received support for this work from the National Institutes of Health (NIH) Award Number K23DA040923. The content of this report is solely the responsibility of the authors and does not necessarily represent the official views of the NIH. The
NIH had no involvement in study design; collection, analysis, and interpretation of data; report writing; or the decision to submit the article for publication.

\section{Conflit d'intérêt Aucun.}

Responsabilité éditoriale Cet article a été traité par Dr Hilary P. Grocott, rédacteur en chef, Journal canadien d'anesthésie.

Financement Karsten Bartels a reçu un soutien financier pour ces travaux des National Institutes of Health (NIH), numéro de bourse K23DA040923. Le contenu de cet article reflète exclusivement l'opinion des auteurs et ne représente pas nécessairement le point de vue officiel des National Institutes of Health. Les NIH n'ont pas pris part à la conception de l'étude; à la collecte, à l'analyse et à l'interprétation de données; à la rédaction de l'article; ou à la décision de soumettre cet article pour publication.

\section{References}

1. Abbott NJ, Patabendige AA, Dolman DE, Yusof SR, Begley DJ. Structure and function of the blood-brain barrier. Neurobiol Dis 2010; 37: 13-25.

2. Abbott NJ, Ronnback L, Hansson E. Astrocyte-endothelial interactions at the blood-brain barrier. Nat Rev Neurosci 2006; 7: 41-53.

3. Jin R, Yang G, Li G. Molecular insights and therapeutic targets for blood-brain barrier disruption in ischemic stroke: critical role of matrix metalloproteinases and tissue-type plasminogen activator. Neurobiol Dis 2010; 38: 376-85.

4. Miller DS, Bauer B, Hartz AM. Modulation of P-glycoprotein at the blood-brain barrier: opportunities to improve central nervous system pharmacotherapy. Pharmacol Rev 2008; 60: 196-209.

5. Potschka H, Fedrowitz M, Loscher W. Multidrug resistance protein MRP2 contributes to blood-brain barrier function and restricts antiepileptic drug activity. J Pharmacol Exp Ther 2003; 306: 124-31.

6. Cheng $Z$, Liu $H, Y u N$, et al. Hydrophilic anti-migraine triptans are substrates for OATP1A2, a transporter expressed at human blood-brain barrier. Xenobiotica 2012; 42: 880-90.

7. Lin CJ, Tai Y, Huang MT, et al. Cellular localization of the organic cation transporters, OCT1 and OCT2, in brain microvessel endothelial cells and its implication for MPTP transport across the blood-brain barrier and MPTP-induced dopaminergic toxicity in rodents. J Neurochem 2010; 114: 717-27.

8. Balyan R, Zhang X, Chidambaran V, et al. OCT1 genetic variants are associated with postoperative morphine-related adverse effects in children. Pharmacogenomics 2017; 18: 621-9.

9. Tzvetkov MV, dos Santos Pereira JN, Meineke I, Saadatmand AR, Stingl JC, Brockmoller J. Morphine is a substrate of the organic cation transporter OCT1 and polymorphisms in OCT1 gene affect morphine pharmacokinetics after codeine administration. Biochem Pharmacol 2013; 86: 666-78.

10. Meissner K, Avram MJ, Yermolenka V, Francis AM, Blood J, Kharasch ED. Cyclosporine-inhibitable blood-brain barrier drug transport influences clinical morphine pharmacodynamics. Anesthesiology 2013; 119: 941-53.

11. Bartels K, Ma Q, Venkatraman TN, et al. Effects of deep hypothermic circulatory arrest on the blood brain barrier in a cardiopulmonary bypass model - a pilot study. Heart Lung Circ 2014; 23: 981-4.

12. Roberts DJ, Goralski KB. A critical overview of the influence of inflammation and infection on P-glycoprotein expression and 
activity in the brain. Expert Opin Drug Metab Toxicol 2008; 4: 1245-64.

13. Dazert P, Suofu $Y$, Grube $M$, et al. Differential regulation of transport proteins in the periinfarct region following reversible middle cerebral artery occlusion in rats. Neuroscience 2006; 142 : 1071-9.

14. Brzica H, Abdullahi W, Ibbotson K, Ronaldson PT. Role of transporters in central nervous system drug delivery and bloodbrain barrier protection: relevance to treatment of stroke. J Cent Nerv Syst Dis 2017; 9: 1179573517693802.

15. Wang Y, Goralski KB, Roberts DJ, et al. An observational study examining the effects of a surgically induced inflammatory response on the distribution of morphine and its metabolites into cerebrospinal fluid. Can J Anesth 2017; 64. DOI: 10.1007/ s12630-017-0933-x.

16. Schinkel AH, Wagenaar E, Mol CA, van Deemter L. Pglycoprotein in the blood-brain barrier of mice influences the brain penetration and pharmacological activity of many drugs. $\mathbf{J}$ Clin Invest 1996; 97: 2517-24.

17. Wandel C, Kim R, Wood M, Wood A. Interaction of morphine, fentanyl, sufentanil, alfentanil, and loperamide with the efflux drug transporter P-glycoprotein. Anesthesiology 2002; 96: 91320.

18. Sadeque AJ, Wandel C, He H, Shah $S$, Wood AJ. Increased drug delivery to the brain by P-glycoprotein inhibition. Clin Pharmacol Ther 2000; 68: 231-7. 